The six trisectors of each of the angles of a triangle.

By F. Glanville Taylor and W. L. Marr.

(Received and Read 14th November 1913.)

1. The following is an account of a theorem whose origin has been traced to Prof. Morley of Johns Hopkins University.

In the course of certain vector analysis, some 14 years ago, Prof. Morley found that if a variable cardioide touch the sides of a triangle the locus of its centre, that is, the centre of the circle on which the equal circle rolls, is a set of 9 lines which are three by three parallel, the directions being those of the sides of an equilateral triangle. The meets of these lines correspond to double tangents; they are also the meets of certain pairs of trisectors of the angles, internal and external, of the first triangle. This result was never published, and it was only the particular case of the internal trisectors that reached the present writers, the existence of the enveloping cardioides and the set of 9 lines being quite unknown to them.

It was only after the present paper had been read that the above information, thanks to various correspondents, was obtained. Hence the theorem has been approached from quite a different point of view, as also the extension of the particular case, which was erroneously thought to be unknown. Certain other properties which follow are, however, probably new.

The particular case will be given first, one proof being due to Mr W. E. Philip, H.M.I.S., from whom the theorem was originally obtained.

2. Morley's Theorem. If the angles of any triangle $A B C$ be trisected, the triangle $D E F$, formed by the meets of pairs of trisectors, each pair being adjacent to the same side of $A B C$, is equilateral. 
First geometrical proof (by W. E. Philip).

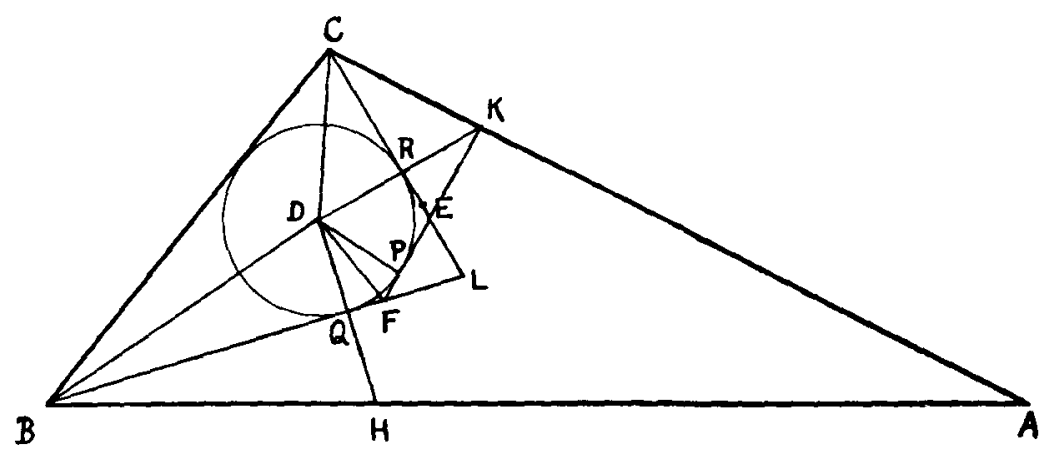

Fig. 1.

Let $\alpha, \beta, \gamma$ stand for $\frac{\mathbf{A}}{3}, \frac{\mathbf{B}}{3}, \frac{\mathbf{C}}{3}$, respectively, so that $\alpha+\beta+\gamma=\frac{\pi}{3}$. The pairs of trisectors of $\mathrm{B}$ and $\mathbf{C}$ meet in $\mathrm{D}$ and L. Draw the incircle of LBC, centre $D$. Let $H, K$ be the images of $\mathrm{D}$ in $\mathrm{BL}$ and $\mathrm{CL}$. Draw the tangent $\mathrm{KP}$ and produce to meet $\mathrm{BL}$ in $\mathrm{F} ; \mathrm{Q}$ is the point of contact of $\mathrm{BL}$.

$$
\text { Since } \begin{aligned}
\mathrm{DP}=\frac{1}{2} \mathrm{DK}, \quad \therefore \quad \angle \mathrm{PKD} & =\frac{\pi}{6} \\
\angle \mathrm{PDK} & =\frac{\pi}{3} \\
\mathrm{QDR}=\pi-\mathrm{L}=2 \beta+2 \gamma & =\frac{2 \pi}{3}-2 \alpha \\
\therefore \quad \mathrm{FHQ}=\mathrm{FDQ}=\frac{1}{2} \mathrm{QDP} & =\frac{1}{2}\left(\mathrm{QDR}-\frac{\pi}{3}\right) \\
& =\frac{\pi}{6}-\alpha .
\end{aligned}
$$

Also

whence

$$
\mathrm{DHK}=\mathrm{DKH}=\frac{1}{2} \mathrm{~L}=\frac{\pi}{6}+\alpha
$$

Hence HFK $=\pi-3 \alpha=\pi-A$, and AHFK is cyclic.

$\therefore \quad \mathrm{HAF}=\mathrm{HKF}=a$, and $\mathrm{AF}$ is a trisector of $A$. Similarly for $\mathrm{E}$, the meet of $\mathrm{CL}$ and the tangent from $\mathrm{H}$. 
From the symmetry of the figure DHK with respect to the circle it follows that $\mathrm{DF}=\mathrm{DE}$ and $\angle \mathrm{FDE}$ is easily seen to be $\frac{\pi}{3}$. $\therefore \triangle \mathrm{DEF}$ is equilateral.

3. Second geometrical proof.

(i) Let $\mathrm{AD}, \mathrm{BE}, \mathrm{CF}$ meet $\mathrm{BC}, \mathrm{CA}, \mathrm{AB}$ in $\mathrm{X}, \mathrm{Y}, \mathrm{Z}$ respectively, also let $X_{1}, Y_{1}, Z_{1} ; X_{2}, Y_{2}, Z_{2} ; X_{3}, Y_{3}, Z_{3}$ be the feet of perpendiculars from $D, E, F$ respectively to the sides of $A B C$.

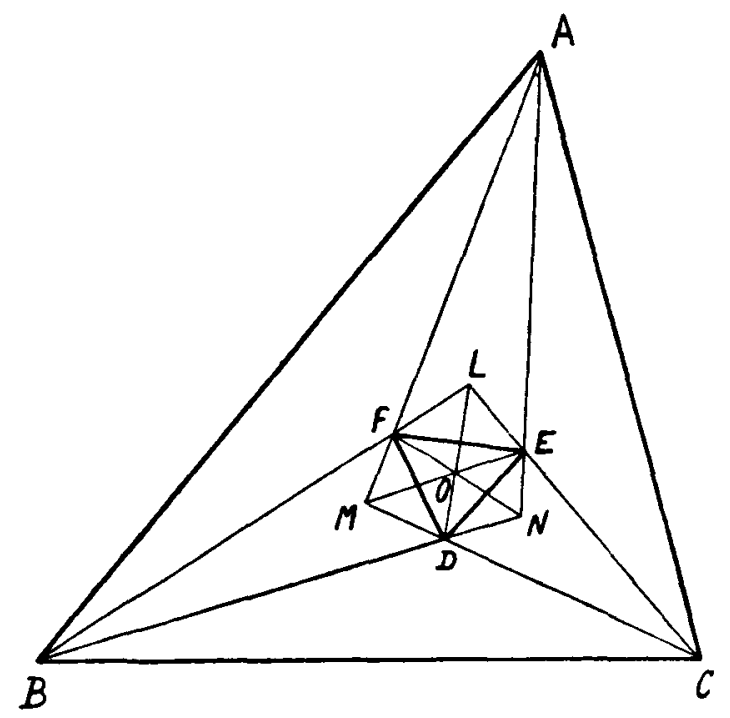

Fig. 2.

Then $\frac{\mathrm{BX}}{\mathrm{XC}} \cdot \frac{\mathrm{CY}}{\overline{\mathrm{YA}}} \cdot \frac{\mathrm{AZ}}{\mathrm{ZB}}=\frac{\triangle \mathrm{ADB}}{\triangle \mathrm{ADC}} \cdot \frac{\mathrm{BEC}}{\mathrm{BEA}} \cdot \frac{\mathrm{CFA}}{\mathrm{CFB}}$

$$
=\frac{\mathrm{DZ}_{1}}{\mathrm{DY}_{1}} \cdot \frac{\mathrm{EX}}{\mathbf{E Z _ { 2 }}} \cdot \frac{\mathrm{FY}_{2}}{\mathrm{FX_{3 }}}=1 \text {, since by similar triangles }
$$

$\frac{\mathrm{EZ}_{2}}{\mathrm{EY}_{2}}=\frac{\mathrm{FY}_{3}}{\mathrm{FZ}_{3}} ; \frac{\mathrm{FX}_{3}}{\mathrm{FZ}_{3}}=\frac{\mathrm{DZ}_{1}}{\mathrm{DX}_{1}} ; \frac{\mathrm{DY}_{1}}{\mathrm{DX}_{1}}=\frac{\mathrm{EX}_{2}}{\mathrm{EY}_{2}} . \quad[\mathrm{Or}$, by Trilinears. $]$

Hence AD, BE, CF are concurrent. 
(ii) It is evident that AFBDCE is a Brianchon hexagon; and taking the sides in the orders AMCLBN, LFMDNE respectively, it follows that $\mathrm{AL}, \mathrm{BM}, \mathrm{CN}$ are concurrent, as also DL, EM, FN. Let the latter point be $O$.

(iii) In the quadrilateral OMDN, since MO and NO bisect the angles at $M$ and $N$, we easily obtain

$$
\angle \mathrm{OMD}=\frac{\pi}{6}+\frac{\mathrm{B}}{3} ; \mathrm{OND}=\frac{\pi}{6}+\frac{\mathrm{C}}{3} \text {; and } \mathrm{MDN}=\frac{2 \pi}{3}+\frac{\mathrm{A}}{3} \text {; }
$$

whence $\mathrm{MON}=\frac{2 \pi}{3}, \mathrm{NOE}=\mathrm{FOM}=\frac{\pi}{3}$.

Hence the acute angles at $O$ are each equal to $\frac{\pi}{3}$, the $\triangle^{\text {les }}$ FOL, EOL are congruent, and LF $=\mathrm{LE}$.

Finally, FDL, EDL are congruent and $\mathrm{DF}=\mathrm{DE}$; whence it follows that $D E F$ is equilateral.

The concurrency of DL, EM, FN may be proved by trilinears thus :-

Since $\mathrm{D}$ is the point $\left(1,2 \cos \frac{\mathrm{C}}{3}, 2 \cos \frac{\mathrm{B}}{3}\right)$, its isogonal conjugate $\mathrm{L}$ is the point $\left(1,1 / 2 \cos \frac{\mathrm{C}}{3}, 1 / 2 \cos \frac{\mathrm{B}}{3}\right)$ or $\left(2 \cos \frac{\mathrm{B}}{3} \cos \frac{\mathrm{C}}{3}, \cos \frac{\mathrm{B}}{3}, \cos \frac{\mathrm{C}}{\mathrm{B}}\right)$. Multiplying the coordinates of $\mathrm{D}$ by $\cos \frac{\mathrm{A}}{3}$ and adding those of $\mathrm{L}$, we get the point (on DL)

$(2 \cos \beta \cos \gamma+\cos \alpha, 2 \cos \gamma \cos \alpha+\cos \beta, 2 \cos \alpha \cos \beta+\cos \gamma)$, where $\alpha=\frac{A}{3}$, etc.

By symmetry it is seen that this point lies on EM and FN ; it is therefore the point $O$.

4. Proof by Trigonometry.

$$
\begin{aligned}
& \frac{\sin \mathrm{AEF}}{\sin \mathrm{AFE}}=\frac{\mathrm{AF}}{\mathrm{AE}}=\frac{c \sin \beta}{\sin \left(\frac{\pi}{3}-\gamma\right)} \cdot \frac{\sin \left(\frac{\pi}{3}-\beta\right)}{b \sin \gamma}=\frac{\sin \left(\frac{\pi}{3}+\gamma\right)}{\sin \left(\frac{\pi}{3}+\beta\right)} . \\
& \text { But } \frac{\pi}{3}+\beta+\frac{\pi}{3}+\gamma=\pi-\alpha=\mathrm{AFE}+\mathrm{AEF}, \\
& \therefore \mathrm{AFE}=\frac{\pi}{3}+\beta ; \mathrm{AEF}=\frac{\pi}{3}+\gamma .
\end{aligned}
$$


Similarly $\mathrm{BFD}=\frac{\pi}{3}+\alpha ; \mathrm{BDF}=\frac{\pi}{3}+\gamma$; etc.

Hence $\mathrm{DFE}=\frac{\pi}{3}$, and similarly for the other angles of DEF. Also $\mathbf{E F}=\frac{\mathrm{AF} \sin \alpha}{\sin \left(\frac{\pi}{3}+\gamma\right)}=\frac{2 \mathrm{R} \sin C \sin \beta}{\sin \left(\frac{\pi}{3}-\gamma\right)} \cdot \frac{\sin \alpha}{\sin \left(\frac{\pi}{3}+\gamma\right)}=8 \mathrm{R} \sin \alpha \sin \beta \sin \gamma$.

The angle $(\mathrm{BC}, \mathrm{FE})=\mathrm{BCA}+\mathrm{CAE}-\mathrm{AEF}$, negatively

$$
\begin{aligned}
& =\mathrm{C}+\alpha-\frac{\pi}{3}-\gamma \\
& =2 \gamma+\alpha-(\alpha+\beta+\gamma)=\gamma-\beta .
\end{aligned}
$$

Hence the directions of FE, DF, ED make angles $\beta-\gamma, \gamma-\alpha$, $\alpha-\beta$ with the sides $\mathrm{BC}, \mathrm{CA}, \mathrm{AB}$ respectively.

\section{Extension of theorem.}

Suppose now we take a more general view of the triangle $\mathrm{ABC}$. Let the angles be $\mathrm{A}+2 p \pi, \mathrm{B}+2 q \pi, \mathrm{C}+2 r \pi$. The angles $\mathrm{BAF}, \mathrm{CAE}$ will be measured in the counterclockwise and clockwise directions respectively, and similarly for the angles at $B$ and $C$. Thus a rotation of the sides inwards such as $\mathrm{BC}$ to $\mathrm{BD}, \mathrm{CB}$ to $\mathrm{CD}$ will be in the positive direction.

Let $\frac{\mathrm{A}}{3}+\frac{2 p \pi}{3}$ be represented by $\alpha_{p}$, etc. Then for each of the six original lines $\mathrm{BD}, \mathrm{CD}$, etc., we shall have three, giving 6 at each vertex. There will be 9 points $D$, and 27 in all. The general triangle will be denoted by $\mathrm{D}_{q r} \mathrm{E}_{r p} \mathrm{~F}_{p q}$ or $[p q r]$, where $p, q, r$ take any of the values $0,1,2$. We shall show that the 27 points D, E, F lie six by six on 9 straight lines, which are 3 by 3 parallel, and inclined at $60^{\circ}$. We thus get 27 equilateral triangles, of which 18 correspond to $D_{00} E_{00} F_{00}$, the remaining 9 involving $D$ 's, E's or F's only. There are also 9 non-equilateral triangles DEF which are closely allied to the 18 referred to above.

$D_{q r}$ then, denotes the point whose biangular coordinates referred to $\mathrm{B}$ and $\mathrm{C}$ are $\left(\beta_{q}, \gamma_{r}\right)$. It will be helpful to remember that $q$ refers to the left vertex $\mathrm{B}$, and $r$ to the right vertex $\mathrm{C}$, as we face BC, etc. [See Fig. 3.]

The coordinates of $\mathrm{D}_{00}$ are $\left(1,2 \cos \gamma_{0}, 2 \cos \beta_{0}\right)$ which we shall denote by $\left(1 \gamma_{0} \beta_{0}\right)$. Hence we easily arrive at the following table 
for $D_{q r}$ or $\left(1,2 \cos \gamma_{r}, \cos \beta_{q}\right)$, or $\left(l \gamma_{r} \beta_{q}\right)$, from which we can deduce $\mathbf{E}_{r p}, \mathbf{F}_{p q}$

\begin{tabular}{c|c|c}
$\mathrm{D}_{00}\left(1 \gamma_{0} \beta_{0}\right)$ & $\mathrm{E}_{00}\left(\gamma_{0} l \alpha_{0}\right)$ & $\mathrm{F}_{00}\left(\beta_{0} \alpha_{0} l\right)$ \\
$\mathrm{D}_{01}\left(1 \gamma_{1} \beta_{0}\right)$ & $\mathrm{E}_{01}\left(\gamma_{0} l \alpha_{1}\right)$ & $\mathrm{F}_{01}\left(\beta_{1} \alpha_{0} l\right)$ \\
etc. & etc. & etc.
\end{tabular}

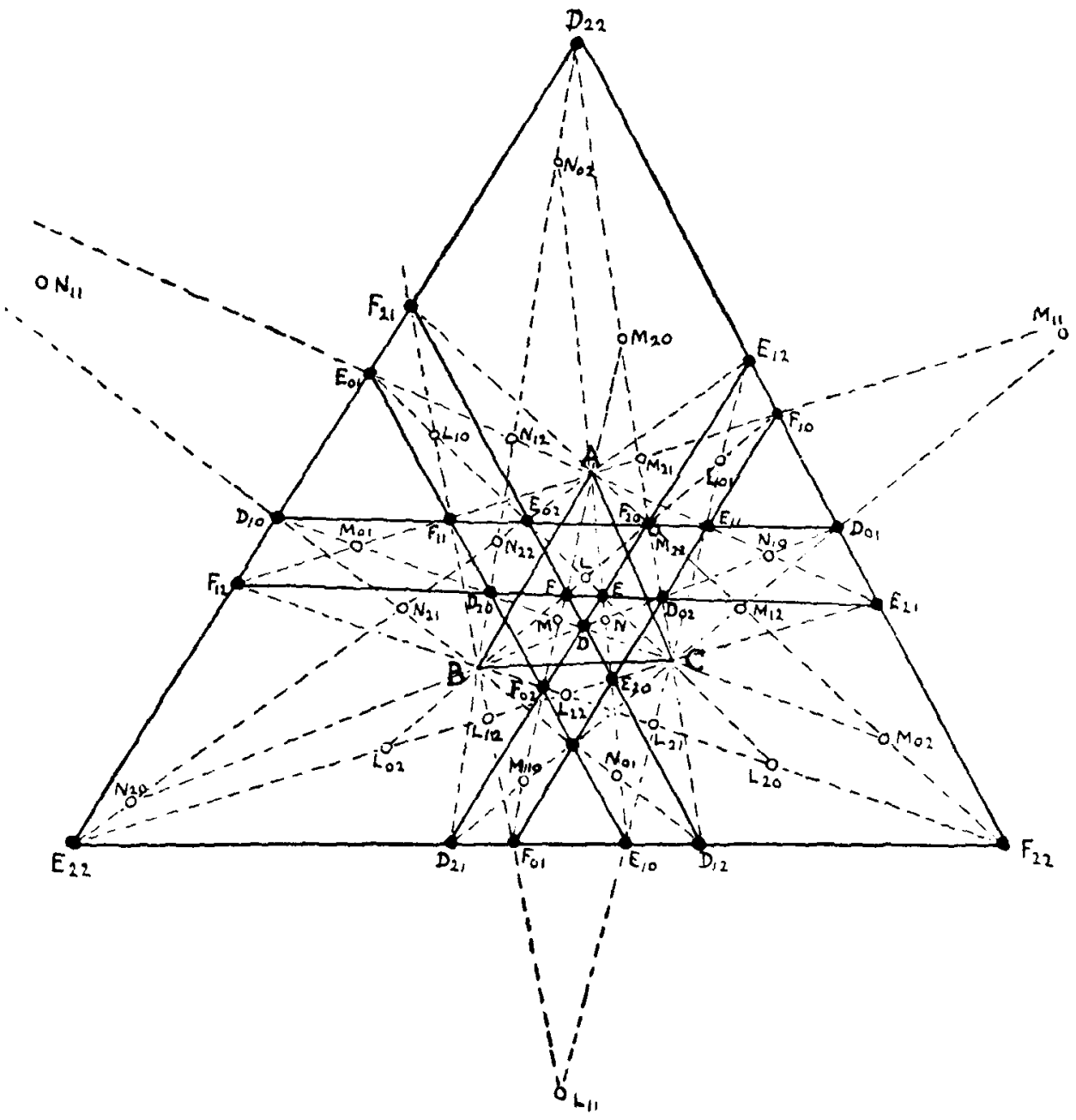

Fig. 3. 
6. The equation to $\mathrm{E}_{00} \mathrm{~F}_{00}$ is reducible to the form

$$
\alpha \sin \alpha_{2}+\beta \sin \beta_{1}+\gamma \sin \gamma_{1}=0
$$

We shall show that besides $E_{00}$ and $F_{00}$ there are four other points $\mathrm{D}_{029}, \mathrm{~F}_{12}, \mathrm{D}_{200}, \mathrm{E}_{21}$ on this same line. We might, of course, merely substitute coordinates, but this is tedious and does not enable us to discover the points. We shall, instead, interchange the letters, increasing and diminishing suffixes so that the sum of the angles remains equal to $\alpha_{2}+\beta_{1}+\gamma_{1}$ or differs from it by $2 \pi$ : also we shall make cyclical changes so as to bring the equation back to its original form. In the case of points on the line a corresponding cyclical change is made in the coordinates including the order as well as the letter.

Thus from $\mathbf{E}_{00}\left(\gamma_{0} 1 \alpha_{0}\right)$ we obtain $\mathbf{F}_{00}\left(\beta_{0} \alpha_{0} 1\right)$ by interchanging $\beta$ and $\gamma$ and the order of the $\beta$ and $\gamma$ coordinates $\left(1 \alpha_{0}\right)$, the equation (1) remaining unaltered by the change.

Next change $\alpha_{p}, \beta_{q}$ to $\alpha_{p-1}, \beta_{q+1}$ respectively, and make a negative cyclical change in $\alpha, \beta, \gamma$. Thus (1) becomes itself again; and the point $\left(\gamma_{0} l a_{0}\right)$ becomes first $\left(\gamma_{0} l \alpha_{2}\right)$ since $2 \equiv-1$ (mod. 3), and afterwards, making a negative cyclical change in the order as well as in the letters, $\left(1 \gamma_{2} \beta_{0}\right)$, which is $D_{02}$.

Interchanging $\beta$ and $\gamma$ we obtain $\left(1 \gamma_{0} \beta_{2}\right)$ or $D_{20}$.

Similarly by changing $\alpha_{p}, \gamma_{r}$ to $\alpha_{p-1}, \gamma_{r+1}$ respectively in (1), and making a positive cyclical change in the letters and their order we obtain $\left(\beta_{3} \alpha_{1} l\right)$ or $F_{12}$; and finally $\left(\gamma_{2} l \alpha_{1}\right)$ or $E_{21}$.

Hence there are six points on $\mathrm{E}_{00} \mathrm{~F}_{00}$, and similarly for $\mathrm{F}_{\infty 0} \mathrm{D}_{\infty}$ and $\mathrm{D}_{00} \mathrm{E}_{00}$. The other sets of six are easily obtained by increasing all the suffixes by 1 , and again by 1 .

Thus $\mathbf{E}_{11} \mathbf{F}_{11}$ is $\alpha \sin \alpha_{0}+\beta \sin \beta_{2}+\gamma \sin \gamma_{2}=0$

$$
\mathrm{E}_{22} \mathrm{~F}_{22} \text { is } \alpha \sin \alpha_{1}+\beta \sin \beta_{0}+\gamma \sin \gamma_{0}=0
$$

In (2) the sum of the angles is unaltered, and in (3) it is diminished by $2 \pi$.

It can be shown by elementary geometry that these three lines are parallel. For example, the triangles $\mathrm{AEC}, \mathrm{ABF}_{01}$ are similar, as also are $\mathrm{AFB}, \mathrm{ACE}_{10}$; hence $\frac{\mathrm{AE}}{\mathrm{AC}}=\frac{\mathrm{AB}}{\mathrm{AF}_{01}}, \frac{\mathrm{AF}}{\mathrm{AB}}=\frac{\mathrm{AC}}{\mathrm{AE} \mathrm{E}_{10}} ; \quad \therefore \frac{\mathrm{AE}}{\mathrm{AF}}=\frac{\mathrm{AE}_{10}}{\mathrm{AF}_{01}}$ and $\mathrm{EF}$ is $\|$ to $\mathrm{E}_{10} \mathrm{~F}_{01}$, etc.

7. To each of the 9 D's correspond three triangles $\mathrm{DEF}$, of which only two, however, are equilateral. Thus keep $\mathrm{D}_{00}$ fixed; 
then since $\beta_{0}$ and $\gamma_{0}$ are fixed the lines BL, CL are fixed. Change $\alpha_{0}$ to $\alpha_{1}$ and $\alpha_{2}$ in succession, i.e. rotate $\mathbf{A E}$ and $\mathbf{A F}$ (in their proper directions, see Art. 5) through $\frac{2 \pi}{3}$ twice in succession and we shall obtain the triangles $\mathrm{DE}_{01} \mathrm{~F}_{10}$ and $\mathrm{DE}_{02} \mathrm{~F}_{20}$. Corresponding to DL, EM, FN we shall have DL, $\mathrm{E}_{01} \mathrm{M}_{01}, \mathrm{~F}_{10} \mathrm{~N}_{10}$ and $\mathrm{DL}, \mathrm{E}_{02} \mathrm{M}_{02}$, $\mathrm{F}_{20} \mathrm{~N}_{20}$. It can be shewn without difficulty that while DL is fixed, EM and FN are turned in opposite directions through $\frac{\pi}{3}$, so that $\mathrm{DL}, \mathrm{E}_{01} \mathrm{M}_{01}, F_{10} N_{10}$ are parallel and the proof breaks down, though not again in the third triangle.

It will be seen that the triangle $[p q r]$ is equilateral provided that $p+q+r \neq 1$ (mod. 3 ), for increasing one of the numbers by unity corresponds geometrically to rotating a pair of the lines $\mathrm{DL}, \mathrm{EM}, \mathrm{FN}$ through $60^{\circ}$ as above. The nine non-equilaterals are $[100],[010],[001],[211],[121],[112],[022],[202],[220]$. Thus [022] is the triangle $D_{22} \mathrm{E}_{20} \mathrm{~F}_{02}$.

The points $L, M, N$.

8. The points $\mathrm{L}, \mathrm{M}, \mathrm{N}$, which are the isogonal conjugates of $D, E, F$, are 27 in number. Noting that the locus of the isogonal conjugates of points on the line $p \alpha+q \beta+r \gamma=0$ is the conic $p \beta \gamma+q \gamma \alpha+r \alpha \beta=0$, 一 the line at infinity, in particular, leading to the circumcircle, - it follows that the 27 points $L, M, N$ lie 6 by 6 on 9 conics through $\mathrm{ABC}$; and that these meet three by three on the circumcircle. It may be shown that the three meeting points form an equilateral triangle whose sides are parallel to those of DEF. We give a short proof :-

Draw through A lines $\mathrm{Ad}, \mathrm{A} e, \mathrm{~A} f$ parallel to $\mathrm{EF}, \mathrm{FD}, \mathrm{DE}$ to meet the circumcircle. Then since the isogonal lines $\mathbf{A} d^{\prime}, \mathbf{A} e^{\prime}, \mathbf{A} f^{\prime}$ make respectively the same angles with $A I$ (I the incentre) as these, but in the opposite sense, it is evident that both def and $d^{\prime} e^{\prime} f^{\prime}$ are equilateral triangles.

$$
\begin{aligned}
& \text { Writing } x \text { for } \gamma_{0}-\beta_{0} \text { or } \frac{\mathbf{C}-\mathbf{B}}{3} \text {, we have, if } x=(d \mathbf{A}, \mathbf{B C}) \text {, } \\
& \angle \mathrm{BAd} d^{\prime}=\mathrm{CAX}=\mathrm{C}-x \quad \text { (Art. 4). } \\
& \mathrm{BAd}=\mathrm{B}+x \text {. } \\
& \therefore \quad d \mathrm{~A} d^{\prime}=\mathrm{C}-\mathrm{B}-2 x=x=\mathrm{AC} d^{\prime} \text {, since } \mathrm{C} d^{\prime} \text { is isogonal to } \\
& \mathrm{C} y(\| \mathrm{AX}), \quad=\mathrm{Add}
\end{aligned}
$$

or $d^{\prime}$ is the mid point of the $\operatorname{arc} A d$. 
Hence $e^{\prime} f^{\prime}$ is parallel to $\mathrm{Ad}$ or EF.

Similarly for $f^{\prime} d^{\prime}$ and $d^{\prime} e^{\prime}$.

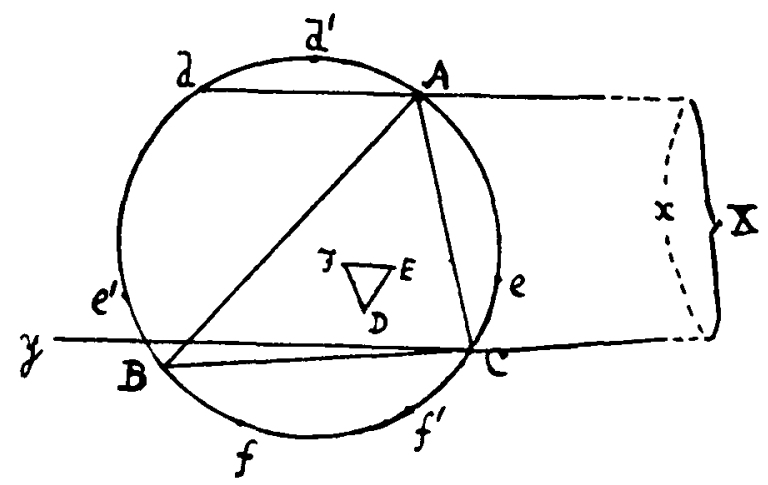

Fig. 4.

9. The lines $\mathrm{AL}_{\mathrm{pm}} \mathrm{BM}_{r p}, \mathrm{CN}_{p y}$ are concurrent; for, referring to the table of Art. 5, we find that $\mathrm{L}_{q}$, the isogonal conjugate of $\mathrm{D}_{q r}$ is $\left(1,1 / \gamma_{r}, 1 / \beta_{q}\right)$ or $\left(2 \cos \beta_{q} \cos \gamma_{r}, \cos \beta_{q}, \cos \gamma_{r}\right)$.

It is thus seen that the point of concurrence is $\left(\cos \alpha_{p}, \cos \beta_{q}\right.$, $\left.\cos \gamma_{r}\right)$. We shall denote this by $(p q r)$.

Corresponding to the 27 triangles DEF are 27 points $(p q r)$.

10. We shall now show (1) that these points $(p q r)$ are the poles of 27 pairs of triangles DEF, comprising the 18 equilateral triangles and the 9 non-equilaterals referred to above; (2) that the former consist of 6 triads of equilaterals, each triad being two by two in perspective; the latter of 3 such triads; and (3) that the poles of each triad are collinear; (4) each line $D_{q r} D_{q+1, r+13}$, etc., passes through three of the points $(p q r)$.

(1) Take a purticular case, $D_{00}\left(12 \cos \gamma_{0} 2 \cos \beta_{0}\right), D_{11}\left(12 \cos \gamma_{1} 2 \cos \beta_{1}\right)$. Multiply the coordinates of $D_{00}$ by $\sin \alpha_{03}$ those of $D_{11}$ by $-\sin \alpha_{1}$, and add.

Then $\sin \alpha_{0}-\sin \alpha_{1}=2 \cos \alpha_{2} \sin \frac{\pi}{3}$.

$2 \cos \gamma_{0} \sin \alpha_{0}-2 \cos \gamma_{1} \sin \alpha_{1}=2 \cos \beta_{0} \sin \frac{\pi}{3}$ eventually,

(see the general case).

$2 \cos \beta_{0} \sin \alpha_{0}-2 \cos \beta_{1} \sin \alpha_{1}=2 \cos \gamma_{0} \sin \frac{\pi}{3}$. 
Hence $D_{00} D_{11}$ passes through (200).

(2) Generally, $\mathrm{D}_{q r}\left(1,2 \cos \gamma_{n}, 2 \cos \beta_{q}\right), \mathrm{D}_{q+1, r+1}\left(1,2 \cos \gamma_{r+1}, 2 \cos \beta_{q+1}\right)$.

Take as multiples $\sin \alpha_{m},-\sin \alpha_{n},(m \neq n)$.

Then (a) $\sin \alpha_{m}-\sin \alpha_{n}=2 \cos \left\{\alpha+(m+n) \frac{\pi}{3}\right\} \sin (m-n) \frac{\pi}{3}$,

(b) $2 \cos \gamma_{r} \sin \alpha_{m}-2 \cos \gamma_{r+1} \sin \alpha_{n}=\sin \left\{\gamma+\alpha+(m+r) \frac{2 \pi}{3}\right\}$

$$
\begin{aligned}
-\sin \left\{\gamma-\alpha+(r-m) \frac{2 \pi}{3}\right\} & -\sin \left\{\gamma+\alpha+(r+1+n) \frac{2 \pi}{3}\right\} \\
+ & \sin \left\{\gamma-\alpha+(r+1-n) \frac{2 \pi}{3}\right\} .
\end{aligned}
$$

Now let $(r+1-n) \frac{2 \pi}{3}-(r-m) \frac{2 \pi}{3}=2 k \pi$.

$$
\therefore m=n+3 k-1 \text {. }
$$

$\therefore \quad$ L.H.S. $=2 \cos \left\{\gamma+\alpha+(m+n+2 r+1) \frac{\pi}{3}\right\} \sin (m-n-1) \frac{\pi}{3}$

$$
\begin{gathered}
=2 \cos \left\{\beta-(2 n+2 r+3 k+1) \frac{\pi}{3}\right\} \sin (3 k-2) \frac{\pi}{3} \\
=2 \cos \left\{\beta-(2 n+2 r+1) \frac{\pi}{3}\right\} \sin \left(-\frac{2 \pi}{3}\right) . \\
=2 \cos \left\{\beta-(n+r-1) \frac{2 \pi}{3}\right\} \sin \frac{\pi}{3}=2 \cos \left\{\beta+(4-n-r) \frac{2 \pi}{3}\right\} \sin \frac{\pi}{3} \\
=2 \cos \beta_{\leftarrow n \rightarrow r} . \sin \frac{\pi}{3} .
\end{gathered}
$$

Similarly $2 \cos \beta_{q} \sin \alpha_{m}-2 \cos \beta_{q+1} \sin \alpha_{n}=2 \cos \gamma_{q-n-q} \cdot \sin \frac{\pi}{3}$.

Also in $(a) \sin \alpha_{m}-\sin \alpha_{n}=2 \cos \left\{a+(2 n+3 k-1) \frac{\pi}{3}\right\} \sin (3 k-1) \frac{\pi}{3}$

$$
\begin{aligned}
=2 \cos \left\{\alpha+(2 n-1) \frac{\pi}{3}\right\} \sin \left(-\frac{\pi}{3}\right)=2 \cos \left\{\alpha+(n+1) \frac{2 \pi}{3}\right\} \sin \frac{\pi}{3} \\
=2 \cos \alpha_{n+1} \cdot \sin \frac{\pi}{3} .
\end{aligned}
$$

Thus $\mathrm{D}_{q r} \mathrm{D}_{q+1, r+1}$ passes through $(n+1,4-n-r, 4-n-q) .^{*}$

* Or, if preferred, $\mathrm{D}_{z-1, r-1} \mathrm{D}_{u}$ passes through the point $(-n, n-r, n-q)$. 
Since $n, q, r$ may have each 3 values, there are 27 points. But there are only 9 lines $\mathrm{D}_{q r} \mathrm{D}_{q+1, r+1}$, since $n$ is not involved here.

We tabulate the results as follows:-

Collinear triads of points.

\begin{tabular}{|c|c|c|c|c|c|}
\hline$q$ & $r$ & $\mathfrak{D}_{g r} \mathrm{D}_{q+1, r+1}$ & $\begin{array}{c}n=0 \\
(1,4-r, 4-q)\end{array}$ & $\begin{array}{c}n=1 \\
(2,3-r, 3-q)\end{array}$ & $\begin{array}{c}n=2 \\
(0,2-r, 2-q)\end{array}$ \\
\hline 0 & 0 & $D_{\infty} D_{11}$ & (111) & $(200)$ & $(022)$ \\
\hline 0 & 1 & $D_{01} D_{12}$ & $(101)$ & $(220)$ & $(012)$ \\
\hline 0 & 2 & $D_{02} D_{10}$ & (121) & $(210)$ & $(002)$ \\
\hline 1 & 0 & $D_{10} D_{21}$ & $(110)$ & $(202)$ & $(021)$ \\
\hline 1 & 1 & $D_{11} D_{22}$ & $(100)$ & $(222)$ & $(011)$ \\
\hline 1 & 2 & $D_{12} D_{20}$ & $(120)$ & $(212)$ & $(001)$ \\
\hline 2 & 0 & $D_{20} D_{01}$ & (112) & (201) & $(020)$ \\
\hline 2 & 1 & $D_{21} D_{02}$ & (102) & (221) & $(010)$ \\
\hline 2 & 2 & $D_{92} D_{\infty 0}$ & (122) & (211) & $(000)$ \\
\hline
\end{tabular}

Similar tables for EE and FF may be obtained merely by a cyclical change in the numbers $(p q r)$, giving $(q r p)$ and $(r p q)$ respectively.

Thus

$$
\begin{array}{ccc}
\mathrm{E}_{20} \mathrm{E}_{01} & \text { gives } & (211) \\
\mathrm{F}_{20} \mathrm{~F}_{01} & " & (121)
\end{array}
$$

11. Since in each of these cases we obtain the same 27 numbers again, but in different orders, it is clear that lines of the type DD, EE, FF are concurrent, as in the following table:- 


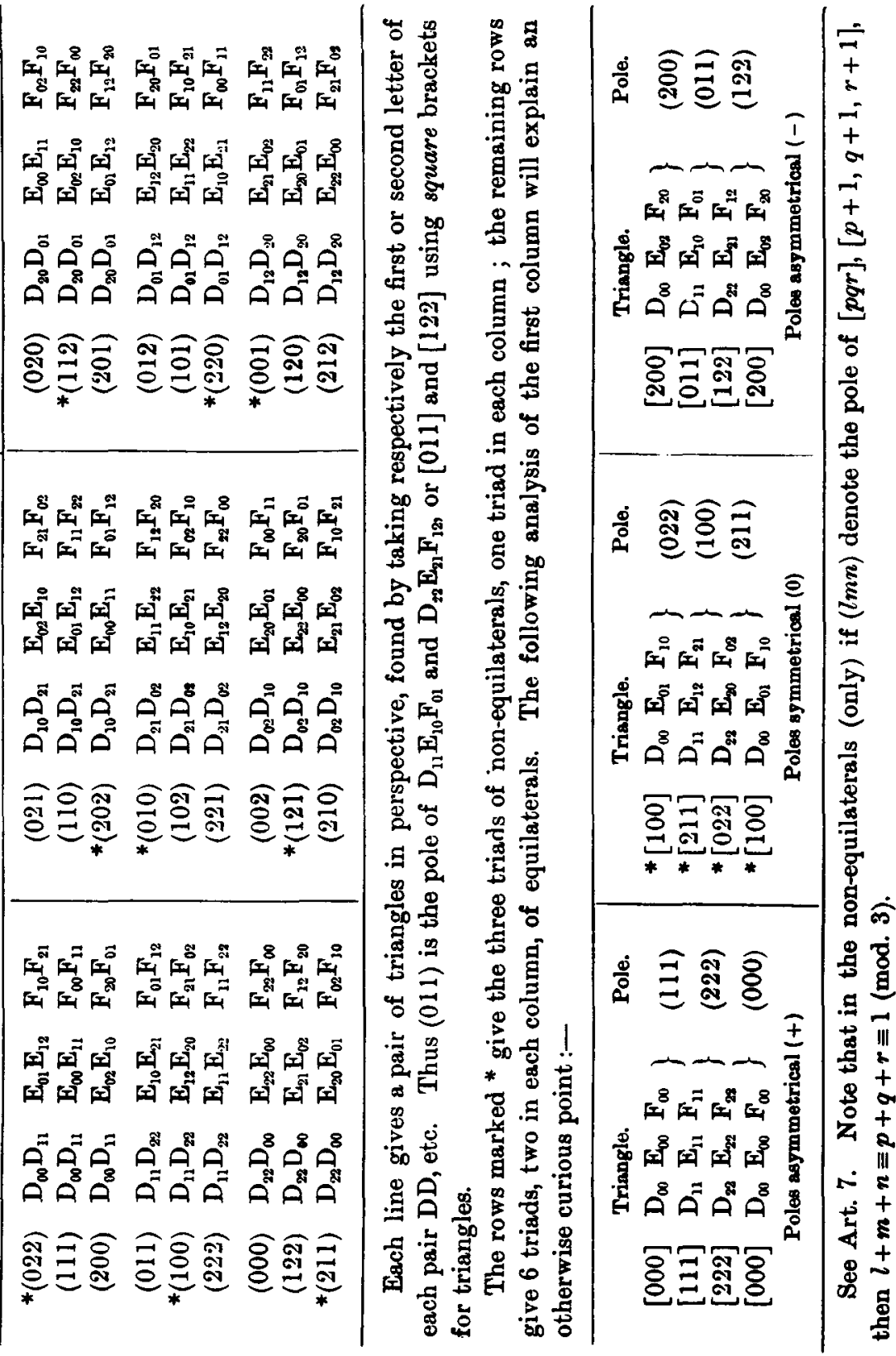




\section{1}

12. The fact that the three poles of any triad of triangles are collinear follows at once from a theorem of Steiner's; for, taking the example given above in the first column, $\mathrm{E}_{00} \mathrm{~F}_{00}, \mathrm{E}_{11} \mathrm{~F}_{11}, \mathrm{E}_{22} \mathrm{~F}_{22}$ meet at the same point on the line at infinity; similarly for the lines FD, DE respectively. Hence the poles of the three pairs of triangles are collinear, as also follows, of course, from the fact that the determinant

$$
\left|\begin{array}{lll}
\cos \alpha_{p} & \cos \beta_{q} & \cos \gamma_{r} \\
\cos \alpha_{p+1} & \cos \beta_{q+1} & \cos \gamma_{r+1} \\
\cos \alpha_{p+2} & \cos \beta_{q+2} & \cos \gamma_{r+2}
\end{array}\right| \equiv 0 .
$$

